\title{
Téoros
}

Revue de recherche en tourisme

\section{Le chemin forestier public, un outil d'éducation}

\section{Claude G. Genest}

Volume 25, numéro 3, automne 2006

Tourisme et forêt, visions d'une gestion intégrée

URI : https://id.erudit.org/iderudit/1071018ar

DOI : https://doi.org/10.7202/1071018ar

Aller au sommaire du numéro

Éditeur(s)

Université du Québec à Montréal

ISSN

0712-8657 (imprimé)

1923-2705 (numérique)

Découvrir la revue

Citer cet article

Genest, C. G. (2006). Le chemin forestier public, un outil d'éducation. Téoros, 25(3), 41-45. https://doi.org/10.7202/1071018ar d'utilisation que vous pouvez consulter en ligne.

https://apropos.erudit.org/fr/usagers/politique-dutilisation/ 


\section{Le chemin forestier public, un outil d'éducation}

\section{Claude G. Genest}

À mon arrivée en Mauricie en 1977, j'ai eu la désagréable surprise de découvrir plusieurs chemins forestiers au début desquels trônait une affiche au message clair : accès interdit, chemin privé, défense d'entrer. Heureusement, le parc national de la Mauricie commençait à peine à ouvrir ses portes ; il y avait donc espoir que le public ait enfin accès à la forêt. Or, le gouvernement du Québec autorisait la construction, depuis plusieurs années, de près de 550 kilomètres de routes forestières par année, pour permettre à l'industrie forestière de collecter les billes de bois. À cette époque et depuis le "déclubage " (début des années 1980), les chemins étaient tenus et gérés comme une exclusivité des compagnies forestières (Genest, 1988 : 107). II a fallu attendre l'arrivée des zones d'exploitation contrôlée (ZEC) pour pouvoir circuler plus librement ou moins dangereusement sur ces chemins forestiers des terres publiques sans risquer d'arriver face à face avec un camion de tiges, chargé à bloc et roulant à grande vitesse (encore aujourd'hui, il faut être muni d'une radio CB pour s'y aventurer). Dans ce bref article, nous traverserons les quinze années de transition vers une plus grande ouverture de la forêt au grand public, donc à la multifonctionnalité et aux multiactivités, par le biais d'une étude de cas : le chemin du Dorval en Mauricie.

\section{Cadre historique}

Le chemin du Dorval, de son vrai nom chemin Joseph-Saint-Amant (section Dorval), se situe dans la municipalité régionale de comté (MRC) de Mékinac. II a la particularité de toucher à trois municipalités, ce qui complique énormément sa gestion. Les cartes anciennes, dont une de 1877, indiquent ce chemin de prélèvement forestier qui débouche sur la rive de la rivière Saint-Maurice, à Saint-Roch-
de-Mékinac. Le chemin est ancien et il a à peine changé de tracé en près de 130 ans. II est toujours là avec son histoire. Un moulin à scie de la famille Frazer a occupé le centre de la vallée dans les années 1940. Géomorphologiquement, le chemin passe dans une faille du plateau des Laurentides dont le fond se compose de multiples plaines d'épandage, de petits deltas suspendus et de quelques ombilics glaciaires, dont le lac Dorval qui donnait anciennement son nom au chemin (Genest et al., 1988). Les parois tapissées de matériaux glaciaires, dont une terrasse de kame, supportent une forêt mixte et diversifiée, typique de l'érablière laurentienne à hêtre, sur les versants regardant vers le sud, et plutôt coniférienne, dont la sapinière à bouleaux jaune et blanc, sur les versants regardant vers le nord (Genest, 1985). Plusieurs autres espèces de conifères et de feuillus tapissent la vallée. Malgré les multiples coupes, peut-être quatre cycles de coupe, les animaux y sont toujours présents, tant la faune ailée que certains mammifères (chevreuil, orignal), le raton laveur et le castor, qui donne son nom à la rivière et qui en occupe le fond. La rivière a ses truites. Les multiples barrages de castors tant sur le cours principal que sur les tributaires donnent un cachet à cette région typiquement " canadienne " dans l'imaginaire européen de "ma cabane au Canada" (Genest, 1997 : 8).

Dans son cadre d'ensemble, l'accès au chemin du Dorval a été facilité par la construction de la route 159 reliant Saint-Tite-de-Champlain et Saint-Roch-de-Mékinac à la fin des années 1930 et permettant de relier le chemin du Dorval à ces deux municipalités. Le dévelop- pement de la villégiature est récent. II s'est effectué dès le "déclubage " au début des années 1980 et s'est étendu sur toute la chaîne de plus de 30 lacs à laquelle le chemin Joseph-Saint-Amant donne accès.

\section{Cas du lac Fontaine}

Dans le cas du lac Fontaine, le développement est plutôt restreint au pourtour du lac qui, par ses escarpements sévères, n'autorise que douze terrains d'une surface de 4000 mètres carrés à peu près, pour chacun des lots. Auparavant, cette chaîne de lacs correspondait au Club Buteux qui gérait, pour des Américains, plusieurs lacs du territoire. Les parois rocheuses témoignent des anciennes marques et annonces de cette institution de chasse et de pêche. Jusque dans les années 1980, la coupe forestière et le Club Buteux cohabitaient. Les activités de coupe forestière présentaient le même profil que dans l'ensemble du Québec. Le bois pour le papier était prélevé par l'AbitibiConsol qui a gardé un terrain long de deux kilomètres en bordure du chemin du Dorval et qui y prélève encore du bois (en 2005). De plus, du bois dur et du bois mou étaient pré- 
levés par des scieries de Saint-Tite (Scierie Joseph-Saint-Amant) et de Saint-Roch-deMékinac (Crête et fils), ainsi que par la scierie temporaire de la famille Fraser (19401948). Les activités à l'époque se déroulaient surtout l'hiver, à part l'entretien des chemins et la construction des camps de bûcherons. Les chasseurs et les pêcheurs en profitaient le reste de l'année en fonction du calendrier d'autorisation des diverses chasses. L'harmonie semblait régner au fil de ces décennies et les habitudes des villages voisins s'adaptaient à ce cycle annuel. Plusieurs personnes travaillaient dans les chantiers, d'autres, ou les mêmes, travaillaient au Club Buteux pour l'entretien ou comme guide de chasse ou de pêche (Genest, 2002).

\section{La période pionnière}

Au lac Fontaine, deux chalets ont pris forme dès le "déclubage " au début des années 1980. Une personne a profité d'un de ses contacts (il était anciennement un des hommes d'entretien du club) pour faire l'acquisition d'un bâtiment du club. Les autres bâtiments ont été rasés. Une autre personne, et son frère, se sont construit un chalet en bois rond au bout du lac. Les travaux de construction sont très méritoires car l'accès y est pratiquement nul. En fait, le problème majeur de l'accès à la forêt publique québécoise se résume à l'état des chemins. Sur des chemins défoncés, cahoteux et rocheux, ils ont construit, à bras d'homme, chacun leur installation en apportant, fin de semaine après fin de semaine, les matériaux, d'où la construction en bois rond au bout du lac, seul matériau disponible. Pour le reste, il leur a fallu transporter en chaloupe les fenêtres, les ferrures, les panneaux et tous les autres objets et outils nécessaires pour construire le chalet. Aujourd'hui nous utilisons le vocable "chalet ", alors qu'à l'époque le terme utilisé était " camp ». C'était un travail de pionnier. En circulant, aujourd'hui, sur le chemin du Dorval, on ne peut qu'imaginer les efforts méritoires de ces pionniers. Cela représente neuf à douze kilomètres de chemin difficile, les derniers kilomètres encore plus difficiles que les premiers (Genest, 1987).

Conséquemment, le chemin c'est l'accès. Accès au chalet, accès à la villégiature en milieu sauvage, naturel. C'est un tout autre contexte que les chalets qui occupent le pourtour de lacs quasi urbanisés comme le lac Beauport ou le lac Saint-Joseph.

\section{La période du développement}

À la fin des années 1980, le ministère des Ressources naturelles (MRN), secteur des Terres, décide de proposer un plan de villégiature pour le lac Fontaine. Le MRN découpe dix autres lots, les officialise par des bornes et les rend accessibles par tirage. Des chalets se construisent par les locataires de ces terrains et futurs résidants en fonction de leurs vacances d'été et de leurs fins de semaine, mais le chemin est toujours aussi mauvais. Les villégiateurs décident, à coup de corvées, de transporter du gravier dans leurs remorques personnelles pour consolider le chemin qui grimpe, en paliers, un versant rocheux de la vallée depuis le talweg jusque sur l'échancrure au sommet de la colline, qui correspond au déversoir du lac. Le lac Fontaine est un lac d'une vallée suspendue, d'où sa difficulté d'accès. De là, le chemin rejoint un ancien chemin de la scierie Frazer, datant des années 1940, mais la forêt a repris sa place après l'abandon des coupes forestières. II leur faut donc couper les nouveaux arbres, enlever les souches, boucher les trous et ajouter du gravier, à bras d'homme et à leurs frais. C'est en 1990 que tous s'assemblent, d'un commun accord, autour d'un des premiers villégiateurs qui possède de la machinerie (bulldozer, pelle mécanique, camion, niveleuse), pour tracer un nouveau chemin plus carrossable que le "sentier des pionniers". Les douze villégiateurs se divisent la facture de 24000 \$ (Genest, 2002).

\section{La problématique du chemin}

Tant que la coupe forestière se réalise sur le territoire de la vallée du ruisseau du Castor, le chemin du Dorval est entretenu, car la société forestière qui a des contrats de coupe à quelques endroits de la vallée veut s'assurer de la qualité du chemin pour le transport des hommes, de la «machinerie " et des matières ligneuses. Faisant partie des derniers arrivants en 1990, je m'aperçois que le chemin est beau, bien entretenu, sans trous ni bosses, et permet une circulation sécuritaire à près de 50 kilomètres/heure sur toute sa longueur, depuis la route asphaltée, la 159, jusqu'à mon terrain sur lequel l'année suivante le chalet est bâti. Cette qualité est maintenue jusqu'en 1994. L'année suivante, le MRN n'autorise pas de contrat de coupe. La dégradation commence. Les trous apparaissent, ainsi que les dommages aux véhicules et la réduction conséquente de vitesse à 30 kilomètres/heure. En 1996, la reprise de la coupe et la construction d'une autre artère fo- restière, la voie $\mathrm{A}$, donnent accès à une autre partie de l'arrière-pays. Le chemin redevient beau, plus praticable. Mais cette période en fait réfléchir plus d'un : qu'arriverait-il s'il n'y avait plus de coupe dans la vallée et ses tributaires? C'est ce qui se passe depuis 1998. Le chemin a été abandonné par les compagnies forestières, les quotas de coupes ayant été atteints dans ce secteur de la Mauricie (Genest, 1988).

En deux années, le chemin s'est vite dégradé. Les pluies de l'automne, la fonte des neiges et les pluies du printemps ont fait que dame nature a érodé le chemin. De plus, les amateurs de véhicule tout-terrain $(V T T)$ et de circulation hors piste s'en sont donnés à cœur joie sur ce chemin dévasté. Devant une telle situation de détérioration, il m'a fallu explorer différentes avenues avec des voisins de chalet afin de restaurer le chemin. Heureusement, en 1999, une société a refait un pont et réparé le chemin pour collecter une petite unité forestière, bien que la réparation ne fut que superficielle. II fallait donc réagir, car les réparations des automobiles, des véhicules à quatre roues motrices (Jeep et autres) et des camionnettes des usagers s'élevaient sans cesse. Seulement à l'été 2002, un rapide relevé m'indique que le total des factures de réparations au garage des douze riverains s'approchait de 17500 \$.

Or, l'entretien du chemin se révèle vite un labyrinthe, car le relevé cadastral indique que le chemin passe sur trois municipalités, mais qu'aucune ne le répare. Après une recherche dans les dossiers municipaux, nous découvrons que le chemin est de niveau provincial et qu'il appartient de fait au ministère des Ressources naturelles, non pas au ministère des Transports. Le MRN a autorisé des compagnies forestières à faire le chemin, c'est-à-dire à le tracer, à soumettre les plans du tracé pour approbation au MRN, puis à couper les arbres, à arracher les souches, à faire le fond de chemin comprenant le drainage (pose de ponts et ponceaux), puis à déposer un tablier de gravier. Un kilomètre de chemin forestier coûtait près de 50000 \$ en 2000. C'est un gros investissement pour une compagnie forestière. Combien plus gros pour des villégiateurs sans ressource! Ces découvertes, jointes à d'autres, dont celles du Volet II, font en sorte que j'ai décidé de former une association à but non lucratif : l'Association des villégiateurs du lac Fontaine. 
Le Volet II est un programme qui permet à des organismes à but non lucratif, dont notre association, d'obtenir des sommes pour améliorer la forêt de différentes façons, par exemple par des plantations, des sentiers d'interprétation, etc. L'état du chemin étant lamentable, l'association table sur sa restauration. Mais, par un concours de circonstances, l'année précédente, un voisin, en collaboration avec la municipalité de Saint-Roch-deMékinac, obtient 24900 \$ pour une première remise en forme d'une portion de 4 kilomètres du chemin. L'année suivante, nous obtenons un autre 25000 \$ pour faire le tour des chemins du lac. En 2004, je fonde l'Association des usagers du chemin Joseph-SaintAmant, qui obtient 25000 \$ pour travailler un tronçon de 4 kilomètres du chemin.

La démarche de restauration est basée sur une vision à long terme qui est d'en faire une vallée éducative et à multiusages. Elle procède d'une réflexion sur la notion de chemin et la multiplicité des activités possibles pour une famille ou des individus.

\section{Réflexion sur la notion de chemin et sensibilisation des villégiateurs}

Le terme chemin mérite une réflexion. Le Petit Robert définit le vocable de plusieurs façons (Robert, 2006 : 415). D'abord dans son point A qui est la première subdivision, l'approche se veut «concrète "; la première définition s'énonce ainsi : «Voie qui permet d'aller d'un lieu à un autre ", avec synonymes: route, voie. Puis, une définition plus spécialisée suit: "Bande déblayée assez étroite qui suit les accidents du terrain (opposé à route, allée) » et qui suggère comme synonymes : piste, sente, sentier. Cela procure un éclairage plus approprié sur la situation contextuelle du chemin étudié. Or à la section $\mathrm{B}$, dont l'approche se veut davantage abstraite, la première définition est la suivante: «1. Distance, espace à parcourir pour aller d'un lieu à un autre", avec synonymes: parcours, route trajet. Plus loin: "2. Direction, voie d'accès", avec synonyme: direction. Puis en 3. : les locutions : «Se mettre en chemin : partir. Poursuivre, passer son chemin, continuer à marcher; ne pas s'arrêter. » Et en 4. : «Trajectoire (d'un mobile). » Plus loin encore le chemin prend un sens métaphorique: "Conduite qu'il faut suivre pour arriver à un but », avec synonymes : moyen, voie. Des exemples sont fournis comme: «il a fait du chemin ", avec les synonymes suivants : aboutir, aller loin, parvenir, progresser, réussir. Finalement: "Se mettre sur le chemin de quelqu'un », avec synonyme: déranger.
Dès le début, le chemin est confiné à la définition première ci-haut : aller d'un point à un autre. II a un caractère essentiellement utilitaire: prendre le bois et l'apporter à l'usine et, cela, depuis les années 1870 jusqu'à maintenant. D'où, pour les usagers qui auront cette perception, souvent les compagnies forestières, les autres usagers dérangent, ils occupent le chemin, ils nuisent à la bonne circulation des camions et des autres appareils.

La deuxième définition, soit la «bande déblayée assez étroite qui suit les accidents du terrain ", correspond, après discussion, à la perception des pionniers qui quittent leur bungalow de banlieue pour aller construire leur "camp ». II est loin d'être une route, il ressemble davantage à une piste, à un large sentier. Le villégiateur pionnier qui a connu des lieux de villégiature analogues au lac Beauport ou au lac Saint-Joseph rêve d'un beau chemin qui rend l'accès plus facile que ce sentier qu'est le chemin Joseph-Saint-Amant en 1980. Après avoir colligé les visions des douze villégiateurs du lac Fontaine, je me suis aperçu que derrière le chemin se situe aussi l'approche métaphorique. Le chemin est un moyen pour chacun de se rendre au chalet, car le chalet c'est « le petit paradis », le lieu d'évasion. II représente souvent, dans l'imaginaire, le calvaire pour atteindre le paradis. C'est aussi, pour certains, une étape de vie, l'accès à un certain "standing » : avoir un chalet » est synonyme de « prestige - parvenir à - atteindre un niveau supérieur ». "Atteindre et parvenir » changent alors d'objet, ils passent du terme chemin au terme chalet, sorte de glissement de sens, et le chemin devient accessoire à l'atteinte du but. Le chalet se présente un peu comme le domaine de l'automobile qui sert à aller d'un point $A$ à un point $B$, mais les psychologues indiquent que c'est aussi un indicateur social; posséder une BMW ou une Mercedes s'associe à l'atteinte d'un niveau social, un autre type de point $B$.

Cet aspect accessoire va devenir un obstacle majeur à la sensibilisation des villégiateurs quant à l'importance du chemin au fur et à mesure que le chemin devient beau: pourquoi s'occuper du chemin puisqu'il est beau? De sorte que le travail sera de concilier le réel et l'imaginaire, par une prise de conscience de l'état du chemin, des bienfaits de son entretien et de l'appropriation par chacun du chemin.

Pour les pionniers, le chemin est essentiel, son entretien comme son amélioration. Ils n'hésitent pas à parler aux visiteurs et aux jeunes qui roulent vite sur le chemin pour les sensibiliser à la vitesse, aux bris et aux accidents que cela peut occasionner. Les villégiateurs qui sont arrivés dans les années 1990, qui n'ont pas connu l'ancien chemin et qui ne circulent donc que par le nouveau chemin, considèrent les travaux antérieurs comme acquis. N'ayant pas connu le passé, ils peuvent difficilement apprécier la différence ; cependant, les années sans entretien leur font penser à ce que pourrait devenir le chemin et cela commence à les préoccuper. Les factures de réparation de leurs divers véhicules facilitent leur prise de conscience.

À l'été 2004, il est donc décidé de faire une association inter-lacs qui s'occupera du chemin Joseph-Saint-Amant. Tous les propriétaires et les locataires de lots qui utilisent le chemin sont convoqués à une réunion dont le cœur porte sur la qualité du chemin. Le premier argument de discussion est la qualité du chemin et les coûts générés pour chacun. L'argument financier personnel a toujours beaucoup d'impact. L'association est créée, j'en suis élu président; mais là ne s'arrête pas l'affaire.

L'étape suivante consiste donc à mettre en place un conseil d'administration pour s'occuper de l'association. Dans cette foulée, je propose une étape supplémentaire: s'approprier psychologiquement le chemin. Nous discutons des endroits à réparer, de la capacité des gens à en faire un peu et, collectivement, nous proposons une session de travail concrète sur le chemin: de l'ébranchage. Pendant la discussion, des membres font signer une pétition aux gens qui passent pour les sensibiliser au mauvais état du chemin et les inciter à devenir membres de l'association. En deux heures l'Association des usagers du chemin Joseph-Saint-Amant voit le jour, au carrefour dudit chemin et de la côte B.

Le conseil d'administration, composé de trois personnes, décide de créer un bulletin de sensibilisation et de valorisation. En outre, je décide de mettre sur pied un bulletin de liaison. Le premier met en valeur la vallée, situe son cadre géologique, géomorphologique, botanique et animalier, puis l'histoire du chemin. Le deuxième montre l'importance d'être en association, la force du nombre et de pouvoir dire son mot dans la gestion du bien commun : le chemin. Le dernier indique les résultats des premiers travaux de l'association avec le montant de 25000 \$ obtenu pour la réfection du chemin et la barrière de dégel. 
En plus des membres et des non-membres du bassin de la vallée de la rivière au Castor, les trois municipalités reçoivent les bulletins, de même que le ministère des Ressources naturelles et la Sûreté du Québec. Des signes témoignent de la prise en charge, comme la mobilisation à l'ébranchage en groupe et, quelques fois, des initiatives personnelles en vue d'améliorer le chemin : déblayage de ponceaux des sédiments accumulés, comblement de trous sur la route, coupe et déplacement d'arbres tombés. C'est une très grosse étape de franchie, car, auparavant, les gens ne se connaissaient pas et vivaient de manière individualiste; aujourd'hui, ils ont conscience de l'importance du chemin et de sa grande fragilité. Avant, les gens se disaient : le gouvernement va s'en occuper. Ils savent maintenant que le gouvernement, même s'il est propriétaire, ne fera rien et que c'est à eux d'y voir. De cette étape de prise en main des villégiateurs découle une étape qui s'adresse au public en général.

\section{Vision du chemin Joseph-Saint-Amant}

Le chemin Joseph-Saint-Amant est un projet qui peut prendre une envergure régionale, car il rend accessible la forêt mauricienne à tous les résidants du Sud du Québec, pour le travail forestier, la récréation et l'éducation. II vise à améliorer la sécurité de passage sur un chemin forestier, d'abord pour les usagers que sont les compagnies qui ont un contrat d'approvisionnement et d'aménagement forestier (CAAF), les employés du gouvernement, les villégiateurs et les amateurs de plein air ; puis, pour le chemin, en améliorant le drainage (clé de la survie du chemin) et le tablier du chemin afin de lui assurer une pérennité ; enfin, pour la nature ellemême, en canalisant les va-et-vient des usagers sans risquer de lui porter atteinte (pollution de l'eau, accident contre un arbre, etc.).

Le projet sera réalisé de façon à favoriser la mise en valeur des ressources forestières. En effet, les interventions proposées permettront de conserver et de protéger les divers potentiels du site : une vallée de faille façonnée par le glacier quaternaire. De plus, les aménagements permettront d'intégrer et de combiner plusieurs activités en un même endroit (exploitation forestière, villégiature, récréation et sports). Dans le futur (dans 3 à 5 ans, peut-être), un volet éducatif à l'aide des panneaux d'interprétation et des sites de piqueniques familiaux sont envisagés, car cette val- lée a beaucoup de potentiel éducatif (géologie, géomorphologie, ornithologie, botanique, mammifères, amphibiens, etc.) et est bien localisée dans la MRC. Plusieurs usagers actuels proviennent de régions comme GrandMère, Shawinigan, Trois-Rivières, Montréal, Drummondville, Repentigny, etc. Dans une optique d'aménagement intégré, notre projet vise à : protéger l'environnement, contrôler l'érosion par un meilleur drainage, améliorer la sécurité des utilisateurs, respecter les limites de la couverture végétale, respecter les lois et les règlements en vigueur, voire à les adapter au milieu - comme les dates de la pêche-, développer la recherche scientifique - déjà des recherches sur l'eau sont en cours par I'Université du Québec à Trois-Rivières, I'Université de Montréal, la Société de géographie de la Mauricie, notamment-, favoriser l'éducation à la nature pour le grand public, tant dans la découverte des nombreuses particularités que dans la protection de la nature. Déjà l'association est alliée au groupe environnemental américain Live No Trace. Des panneaux que nous avons francisés avec leur permission seront discrètement posés sous l'étiquette «sans trace». Nous serons le premier regroupement francophone sans trace.

Les retombées pour la collectivité vont, de façon générale, faciliter l'accès récréotouristique (pêche, chasse, cueillette, canotage, vélo de montagne, baignade, plongée sousmarine, randonnée pédestre, marche sportive, photographie de la nature, équitation, traîneau à chien, motoneige, VTT, ski de fond, observation de piste d'animaux, etc.) ; améliorer la sécurité routière et faciliter l'accès aux intervenants du service 911; favoriser le développement de l'expertise de la construction de chemins forestiers de type récréotouristique; encourager le partenariat de l'Association des usagers du chemin JosephSaint-Amant avec les municipalités de Grandes-Piles, de Saint-Roch-de-Mékinac, de Sainte-Thècle, la MRC de Mékinac et les firmes responsables des CAAF ; renforcer la recherche scientifique du milieu hydro-forestier (la Société de géographie de la Mauricie inc. y fait des études nivales et hydriques, I'Université du Québec à Trois-Rivières y étudie des planctons et l'Université de Montréal procède à des tests de qualité de l'eau) ; favoriser l'éducation du grand public aux sciences naturelles (géologie, géomorphologie, ornithologie, botanique, biologie, etc.). Le grand défi sera de concilier la protection de la nature et l'utilisation anthropique des ressources naturelles.

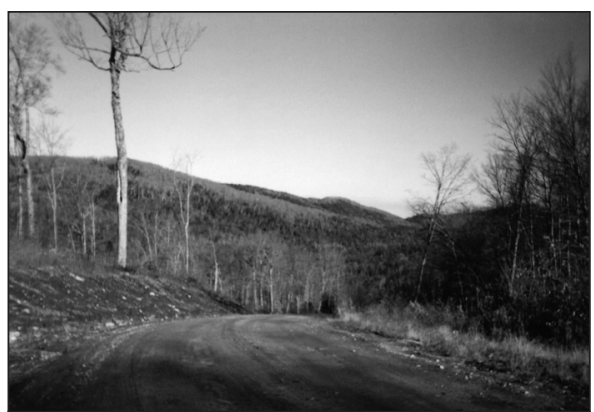

Chemin Joseph-Saint-Amant, haut de la côte $B_{\text {, }}$ avec une vue de la vallée de la rivière du Castor en contre-bas, à la mi-octobre.

Photo Claude G. Genest

L'origine de cette vision est sans doute née de l'absence d'accès pour une famille à des lieux naturels en Mauricie qui regorge pourtant de surfaces forestières pour marcher, pique-niquer, observer la nature et vivre le calme et la détente.

Dans les faits, depuis quinze ans, ce chemin a vu graduellement croître son usage; il est passé de la bivalence coupe forestière-villégiature à un multiusage. Au printemps, les villégiateurs reviennent à leur chalet, les pêcheurs taquinent la truite dès le $1^{\mathrm{er}}$ mai, un club de VIT fait des promenades et rejoint un sentier provincial de VTT près du lac du Jésuite. En été, c'est la haute saison, pour les parents et les amis des villégiateurs. Les membres du club équestre les Quatre Fers y passent avec des voitures tirées par des chevaux et des cavaliers seuls. Les marcheurs se manifestent d'autant plus que la municipalité de GrandesPiles a construit une halte sur le sentier de marche canadien qui passe sur leur territoire et qui est dans le prolongement du chemin Joseph-Saint-Amant, de l'autre côté de la route 159. En automne, c'est la chasse; de nombreux chasseurs, pour différents gibiers selon les périodes attitrées, se logent dans les sentiers de la vallée et attendent leur proie. L'hiver, quelques villégiateurs se rendent en motoneige à leur chalet ; c'est le seul moyen de locomotion, le chemin n'étant pas ouvert. Durant la période sans neige, un éleveur de chiens de traîneau entraîne ses chiens durant la semaine, en période où il y a peu de circulation de véhicules. La période sans feuille du printemps attire de nombreux marcheurs qui aiment se promener hors sentiers et voir loin sans la présence des feuilles. En période végétale, durant la saison la plus chaude, quelques photographes, amateurs de la nature, s'en donnent à cœur joie le long de la route en bordure d'un grand marécage où plusieurs espèces d'oiseaux sont présentes. 
La majeure partie des activités qui y ont cours sont récentes. Par exemple, depuis 1998, I'Université de Montréal prélève des échantillons d'eau à titre d'indicateur témoin de pollution au lac Fontaine; en 2001 l'Université du Québec à Trois-Rivières s'intéresse au plancton et la Société de géographie de la Mauricie et y effectue des mesures de précipitations pluvieuses et de neige. Alors qu'en 1990 il n'y avait que deux VTT, le nombre s'est accru, si bien qu'en 2005, presque $75 \%$ des villégiateurs en ont un, quelques-uns en ont deux. Depuis 2000, les gens de la région viennent stationner leur véhicule près de la route 159 et partent pour la journée se balader en VTT en faisant la tournée de quelques villages. Pour l'instant la cohabitation des activités s'effectue en harmonie, mais c'est un équilibre fragile car il y a un effet de mentalité : les gens non avertis sont portés à croire que le chemin appartient au gouvernement, qu'ils peuvent donc l'utiliser, malheureusement sans aucune considération, y circuler en VTT avec agressivité, faire gicler du gravier à un passant et briser le chemin (observations, été 2005). Actuellement, il reste une signalisation à placer avec minutie et discrétion, présentant des messages simples comme: chemin fragile, chemin patrimonial, chemin entretenu par les usagers et autres termes de sensibilisation. A contrario, les usagers sont devenus sensibles; ils arrêtent pour ramasser des papiers, des arbres ou des branches tombés et d'autres déchets malencontreux, mais le citoyen non averti est malheureusement souvent irrespectueux et se sent chez lui partout. Certains même insultent les villégiateurs lorsque ces derniers tentent de leur parler.

Cette harmonisation des différentes activités a demandé plusieurs rencontres et les usagers ont appris à se connaître et à développer une complicité civique sans donner dans le «gros village » où tout le monde s'intéresse aux affaires des autres («mémérage » ou « cancanage »).

\section{Expérience récente - avril 2006}

Cette année, le conseil d'administration de l'association a décidé de fermer le chemin pour la période de dégel. Les membres ont été informés par le bulletin de l'association et ils doivent se charger d'en avertir parents et amis. Le ministère des Ressources naturelles en a avisé les contractuels de la forêt et la Sûreté du Québec. L'esprit de collaboration a été tel, chez les usagers, que dès la première fin de semaine, quinze véhicules étaient stationnés à l'entrée du chemin. Les usagers ont utilisé leur VTT pour se rendre à leur chalet, soulageant ainsi le chemin du poids de leur véhicule, souvent des camionnettes. C'est une belle réussite, malgré que deux pêcheurs non avertis se soient aventurés de nuit sans avoir vu les affiches. Heureusement, au 30 avril, la surface du chemin était pratiquement sèche, donc il y a eu peu de dégâts.

\section{Conclusion}

Le multiusage de la forêt est possible dans des conditions de dialogue avec les usagers, de développement de leur sensibilisation à la richesse de leur environnement naturel, par différentes méthodes, par exemple un journal qui fait découvrir aux villégiateurs les richesses de leur milieu, des réunions d'information, l'encouragement à participer à des activités communes comme le débroussaillage. Ces quinze dernières années ont été marquées par le passage d'un bi-usage (industrie et chassepêche) vers une ouverture au multiusage. La différence est nette. En 1990, un villégiateur se disant « harcelé » par les pêcheurs a stationné sa camionnette en travers du chemin, fermant ainsi l'accès à un lieu dont il jugeait être le seul bénéficiaire et propriétaire, sorte de vestige de la mentalité des anciens clubs de pêche (Genest, 2003). Or, en 2005, la rampe de mise à l'eau à l'accès public du lac Fontaine a été refaite par les résidants.

Nous nous sommes interrogés sur la manière de transformer un objet strictement utilitaire, un chemin forestier ordinaire, en un objet plus relevé. Dans les faits, nous sommes passés d'un phénomène linéaire reliant deux points à un phénomène spatial, par une prise de conscience des usagers quant aux différentes valeurs des bordures ou des côtés du chemin, voire même les «à-côtés ", soit la récréation en plein air, l'observation de la nature, la protection de certains sites, comme le marécage, et le caractère paysager du chemin. Le chemin a glissé d'une gestion gouvernementale à une gestion entièrement associative. Les usagers ont décrété eux-mêmes des aires à protéger, d'autres à aménager. La participation à la gestion, qui n'existait pas il y a quinze ans, mobilise maintenant les secteurs provincial, puis régional (MRC), municipal et enfin local (les associations présentes sur le terrain). Le chemin est devenu un objet d'étude scientifique. L'approche du chemin, dans le plan de développement, après avoir connu une phase de sensibilisation, se dirige vers l'éducation et vraisemblablement vers l'interprétation par des panneaux idoines se combinant à des aires de pique-niques familiaux.
Claude G. Genest est professeur de géographie à l'Université du Québec à Trois-Rivières.

\section{Bibliographie}

Genest, C. (1985), L'espace rural en bordure du Bouclier canadien, Congrès de l'Association des géographes canadiens, livret-guide d'excursion, Trois-Rivières, Province de Québec, Canada, 28 p.

Genest, C. (1987a), «L'utilisation conflictuelle de la forêt mauricienne ", Annales du Congrès des sociétés savantes, Association canadienne des géographes, Hamilton, Ontario, Canada, p. 23-25.

Genest, C. (1987b) « Le cadre de la difficile gestion de la forêt mauricienne: Intérêts et conflits ", The Operational Geographer / La géographie appliquée, Ottawa, Canada, nº 13, p. 19-22.

Genest, C. (1988), «Les enjeux géographiques de la loi 150 (la forêt), le cas de la Mauricie ", Annales de l'ACFAS, Association canadiennefrançaise pour l'avancement des sciences, Moncton, Canada.

Genest, C. (1989) "Les changements géographiques du nouveau régime de gestion de la forêt du Québec, le cas de la Mauricie", Recueil géographique de Trois-Rivières, TroisRivières, vol. 1, p. 36-45.

Genest, C.G. (1997), L'avenir de la forêt publique en Mauricie, Animateur d'atelier sur les pratiques et usages multiples de la forêt, 20 mai, Hôtel Le Baron, Trois-Rivières, Province de Québec.

Genest, C.G. (2002), « Expérience de villégiature en forêt publique ", Géographes, Bulletin de l'Association professionnelle des géographes du Québec, Montréal, n 12, décembre, p. 34-40.

Genest, C.G. (2003), La villégiature sur les terres publiques, la conciliation des usagers, Panel de l'Association forestière de la Vallée du Saint-Maurice, 26 février, Université du Québec à Trois-Rivières.

Genest, C., J. Beauchesne, C. Brisson, et J. Genest (1988), Répertoire des problèmes environnementaux des municipalités de la région Mauricie-Bois-Francs, Société de géographie de la Mauricie Inc., Trois-Rivières, 215 p.

Genest, C., et Z.I. Offer (1986), "The Junction of the Saint Lawrence and Saint Maurice Rifts: An Ecological Patchwork", dans Z. Dubunsky et $Y$. Steinberger (dir.), Environmental Quality and Ecosystem Stability, vol. III A/B, Bar-Ilan University Press, Ramat-Gan, Israël, p. 429-439.

Robert, Paul (2006), Nouvelle édition du Petit Robert, Dictionnaire Le Robert, Paris, 2949 p. 